\title{
Side effects and flares risk after SARS-CoV-2 vaccination in patients with systemic lupus erythematosus
}

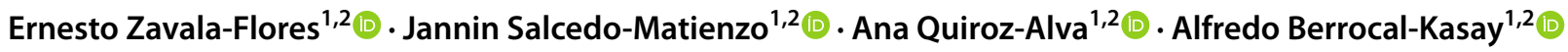

Received: 20 August 2021 / Revised: 18 September 2021 / Accepted: 31 October 2021 / Published online: 16 November 2021

(c) International League of Associations for Rheumatology (ILAR) 2021

\begin{abstract}
The objective of this study is to identify post SARS-CoV-2 vaccine BNT162b2 (BioNTech \& Pfizer) side effects in patients with systemic lupus erythematosus (SLE) at the Cayetano Heredia Hospital, Lima, Peru. A descriptive observational study was designed in patients with SLE at the Immuno-Rheumatology Department of the Cayetano Heredia Hospital, Lima, Peru, immunized with the BNT162b2 vaccine from May 21 to June 30, 2021. Of the total number of patients seen in the service, 100 received the vaccine's 1st dose, and 90 patients received the 2nd dose; $90 \%$ and $92.2 \%$ presented symptoms within 10 days after immunization ( 1 st and 2 nd doses, respectively), being pain at the inoculation site the most frequent (87\%); most of the symptoms presented were of mild intensity. There were 27 episodes of post-immunization flare, $9 \%$ and $20 \%$ after the 1st and 2nd doses, respectively; the predominant type of flare was articular (85.1\%), followed by dermal (18.5\%). It was found that a history of renal involvement was associated with the risk of developing flare RR $0.38(0.15-0.91)$ and the use of hydroxychloroquine and azathioprine prior to immunization $0.20(0.06-0.63)$ and $7.96(2.70-23.43)$ respectively. In 100 SLE patients immunized with BNT162b2 vaccine against SARS-CoV-2, 27\% of SLE reactivation episodes occurred, two patients were hospitalized for flare severity, and none died.
\end{abstract}

Key Points

- Up to $92.2 \%$ presented some type of symptom after vaccination, being mostly local and of mild intensity.

- Of the population studied, there were 27 episodes of post-vaccination flare, most of which were mild.

- In the studied population, taking hydroxychloroquine and having a history of renal disease were associated with a lower risk of presenting post-vaccination flare.

Keywords (MeSH NLM): Coronavirus · Autoimmune diseases · COVID-19 · Systemic lupus erythematosus · Vaccines

\section{Introduction}

To date, the main tools for COVID-19-related mortality are vaccines. The new methods in the development of vaccines (mRNA and viral vectors) initially caused concern in the population given the lack of previous experience with this technology; however, clinical trials showed adequate levels of efficacy and safety in the included population [1-3].

Ernesto Zavala-Flores

ernestozav123@gmail.com

1 Cayetano Heredia Hospital, Av. Honorio Delgado, N²62, San Martin de Porres, Lima, Peru

2 Cayetano Heredia Peruvian University, FMH-UPCH, Lima, Peru
However, these studies did not include patients with autoimmune diseases, which have not made it possible to establish a level of efficacy and/or safety for this group of patients (considering the immunological alteration inherent to these diseases, immunosuppressive therapy, and the possible impact on disease activity) [1, 4-6].

Some studies have documented post-immunization side effects in patients with autoimmune diseases; and in the particular case of SLE, post-immunization episodes of disease reactivation (flare) have been described, as in the case of pneumococcal, influenza, and human papilloma virus vaccines $[7,8]$. However, to date there are limited communications studying the manifestations presented in patients with SLE vaccinated against SARS-CoV-2 [9-12].

The main objective of the present study was to evaluate the manifestations and tolerance following immunization 
with the BNT162b2 vaccine against SARS-CoV-2 in patients with SLE in a public hospital in Peru.

\section{Materials and methods}

A descriptive observational study was designed, where the study population were patients with the diagnosis of SLE attended at the Immuno-Rheumatology Service of the Cayetano Heredia Hospital, Lima, Peru, who were immunized with the BNT162b2 vaccine (BioNTech \& Pfizer) between May 21 and June 30, 2021.

Patients with a diagnosis of SLE were included, according to the classification criteria of SLICC 2012 [13] and/or ACR/EULAR 2019 [14], all older than 18 years, who had received at least one dose of BNT162b2 vaccine (BioNTech $\&$ Pfizer) during the established period. Patients who had been immunized with another type of SARS-CoV-2 vaccine and patients not authorizing telephone follow-up and/ or face-to-face post-immunization evaluation were excluded.

The variables recorded were sex (male-female), age, time of illness, comorbidities, history of documented SARS-Cov- 2 infection, presence of flare within 6 months prior to immunization, type of systemic involvement by SLE, usual treatment for SLE, clinical manifestations following the 1st and 2nd doses of immunization (local reaction, pain at the site of inoculation, headache, fever, fatigue, joint pain, myalgia, nausea, vomiting, abdominal pain, diarrhea), presence of flare after the 1st and/or 2nd dose of immunization (confirmed by a rheumatologist in a face-toface evaluation), and type of flare presented. Flare was defined as a measurable increase in disease activity in one or more organ systems involving clinical or laboratory findings (new or worse) [15], using laboratory parameters (ESR, CRP, 24-h proteinuria, DNAds, serum complement) before and after immunization.

In the case of post-immunization manifestations, symptom intensity was graded as follows: mild, if the symptom did not interfere with daily activities; moderate, if the symptom partially interfered with the normal performance of daily activities; severe, if the symptom prevented the performance of daily activities. For the variable, fever was graded 38.0 to 38.5 (mild), 38.6 to 39 (moderate), and greater than 39 (high).

Procedures As part of routine care, all patients were evaluated face to face prior to SARS-CoV-2 immunization. Subsequently, with their consent, they were followed up within 10 days by telephone. Similarly, in the case of patients with suspected disease reactivation, they were re-evaluated face-to-face to confirm the flare and document the corresponding characteristics.

The present study was approved by the Cayetano Heredia Hospital ethics committee (code 039-2021); the identity of the participants was respected and protected; only the researchers had access to the database, which was encrypted to maintain the confidentiality of the participants.

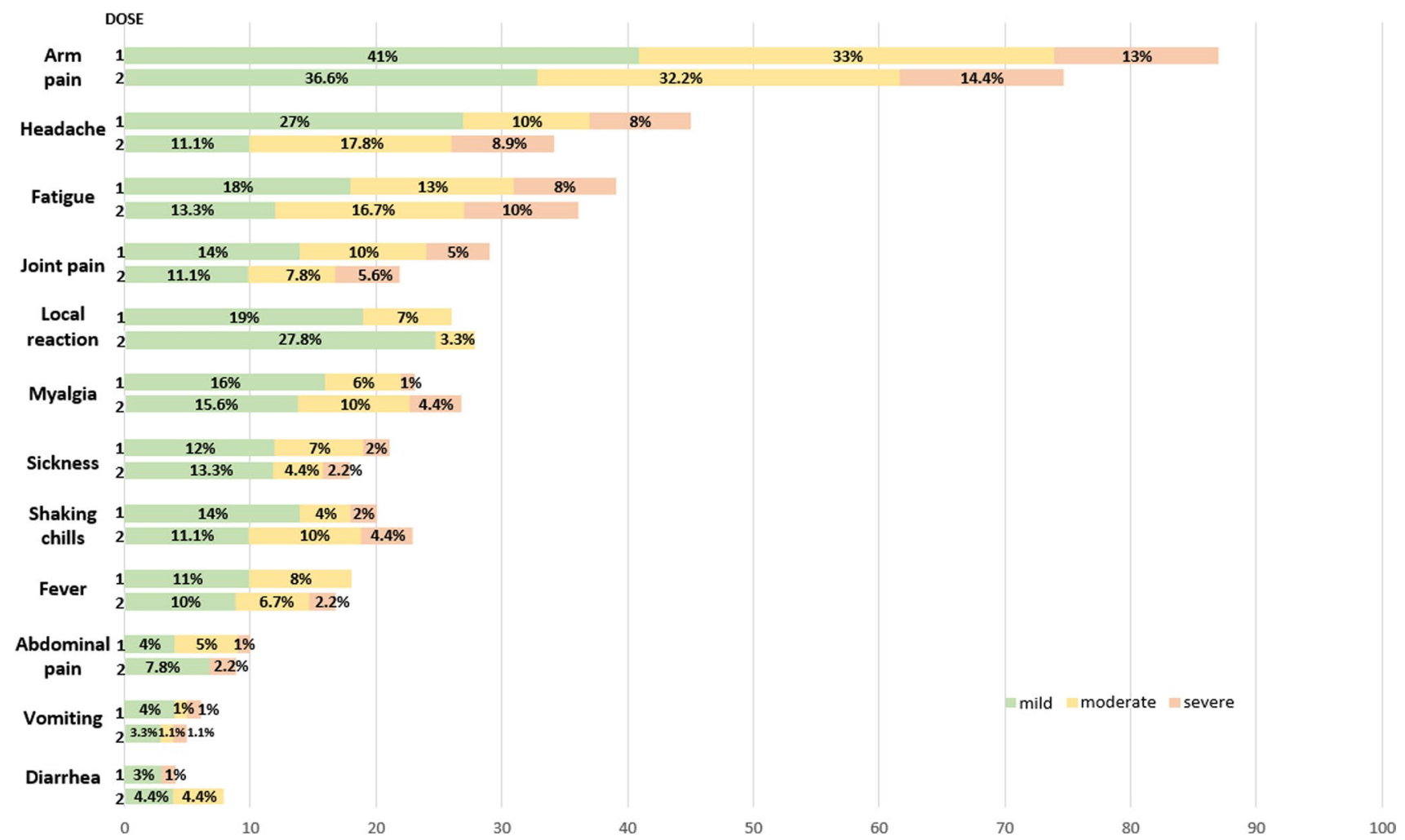

Fig. 1 Symptoms presented in patients with lupus after the 1st and 2nd doses of immunization against SARS-CoV-2 
For the analysis of numerical variables, the mean/standard deviation or median/interquartile range was used for parametric and nonparametric distribution, respectively; for dichotomous variables, absolute and relative frequency was used. For categorical variables, the chi-square or Fisher test was used when any of the assumptions were not met; in both cases, statistical significance was established for a $p$ value $<0.05$ and a $95 \%$ confidence interval $(95 \% \mathrm{CI})$. Covariate adjustment for possible confounding factors was also performed using Poisson regression.

Microsoft Office Excel 2016® and the statistical program STATA v15® were used as computer support.

\section{Results}

A total of 100 patients with SLE immunized with the BNT162b2 vaccine (BioNTech \& Pfizer) were studied; the mean age was 38.9 years, $94 \%$ were women; the most frequent systemic manifestations of SLE were articular $74 \%$, renal $60 \%$, and dermal $23 \%$. The $81 \%$ of patients were taking treatment to control SLE, $28 \%$ presented reactivation of SLE within 6 months prior to immunization (Appendices Table 3 and Figure 2).

Of the total, 100 patients received the 1 st vaccine dose and 90 the 2nd dose; $90 \%$ and $92.2 \%$ presented symptoms within 10 days after immunization (1st and 2nd doses, respectively), with pain at the inoculation site being the most frequent (87\%). Most of the symptoms presented were of mild intensity. On average, these symptoms occurred $1.1 \pm 0.5$ days after vaccination, and the duration was $3.5 \pm 1$ days (Fig. 1).

There were 27 episodes of flare after the immunization process, 9\% (9/100) and 20\% (18/90) after the 1st and 2nd doses, respectively; seven patients presented reactivation in both immunization processes. The predominant type of flare was arthritis (synovitis, swelling, phlogosis) $(85.1 \%)$, followed by dermal (18.5\%) (Table 1, Appendix Table 4). The time of onset of reactivation was $2.3 \pm 0.8$ days; the duration was $7.3 \pm 3$ days; it was found that the history of renal involvement was associated with the risk of developing flare RR 0.38
Table 1 Clinical characteristics of flares post SARS-CoV-2 immunization

\begin{tabular}{ll}
\hline Total & $20^{\mathrm{a}}$ \\
\hline Age-years & $38.65 \pm 14.13$ \\
Sex-no. (\%) & \\
Female & $18(90 \%)$ \\
Male & $2(10 \%)$ \\
Length of illness (SLE)-years & $7.04 \pm 4.85$ \\
SLE treatment & \\
Prednisone & $16(80 \%)$ \\
Hydroxychloroquine & $11(55 \%)$ \\
azathioprine & $10(50 \%)$ \\
Mycophenolate & $3(15 \%)$ \\
Methotrexate & $3(15 \%)$ \\
Leflunomide & $1(5 \%)$ \\
No treatment & $3(15 \%)$ \\
SLE outbreak in the past 6 months & $8(40 \%)$ \\
Confirmed COVID-19 history & $13(65 \%)$ \\
Flare episodes after immunization & \\
1st dose & $9(45 \%)$ \\
2nd dose & $18(90 \%)$ \\
1st and 2nd doses & $7(35 \%)$ \\
& Type of flare $1 \mathrm{era} \mathrm{dosis}$ \\
Arthritis & $6(30 \%)$ \\
Malar erythema & $1(5 \%)$ \\
Alopecia & - \\
Lupus pneumonitis & $1(5 \%)$ \\
Mykopenia & $1(5 \%)$ \\
Hospitalization for flare & $1(5 \%)$ \\
\hline & 2 \\
\hline
\end{tabular}

${ }^{a} 20$ patients presented post-immunization flare; however, there were 27 flare episodes (given that 7 patients had flare episodes in the 1 st and 2 nd doses)

${ }^{\mathrm{b}} 15$ patients use 2 or more drugs 
(0.15-0.91) and the use of hydroxychloroquine and azathioprine prior to immunization $0.20(0.06-0.63)$ and 7.96 (2.70-23.43), respectively (Table 2).

\section{Discussion}

Infections are among the main causes of morbidity and mortality in patients with SLE; therefore, vaccination is advised (especially vaccines originating from non-living microorganisms) [16]. While the benefit of immunization against infections in SLE patients is undeniable, it should also be noted that isolated cases of post-immunization flare have been reported in the literature [7, 8]. In the case of SARSCoV-2 vaccines, especially mRNA and viral vectors, theoretical mechanisms have been proposed that could lead to a possible SLE reactivation (production of interferon alpha as well as a probable molecular mimicry of the SARS-CoV-2 Spike protein) $[2,3]$.
In a study of adverse reactions associated with the SARS-CoV-2 vaccine (Pfizer/BioNTech and Moderna) in systemic autoimmune diseases [5], it was found that $89 \%$ presented local symptoms and 69\% systemic symptoms (mainly fatigue, headache, myalgia). Boekel [11] reported that in patients with systemic autoimmune diseases vaccinated mainly with Pfizer/BioNTech and AstraZeneca, 51\% presented mild adverse effects and $21 \%$ moderate effects. In our study, $87 \%$ presented local symptoms (pain at the inoculation site), and $69 \%$ presented systemic symptoms (mainly headache, fatigue, joint pain, and myalgias) in both the 1st and 2 nd doses of immunization.

A possible relationship between the SARS-CoV-2 vaccine and autoimmunity has been described in observational studies. Watad et al. [10] reported a series of 27 patients, of whom 17 presented reactivation of their baseline autoimmune disease and 10 presented debut of autoimmunity after SARS-CoV-2 immunization. In our study, we found 27 flare episodes (clinical and/or laboratory worsening) after immunization ( 9 and
Table 2 Associated factors to flare after SARS-COV-2 immunization

\begin{tabular}{|c|c|c|c|c|}
\hline & Flare & No flare & $P$ value $^{\mathrm{a}}$ & RR-IC \\
\hline $\begin{array}{l}\text { Age, years } \\
\text { Median (IQR) }\end{array}$ & $\begin{array}{l}37 \\
(28-43.5)\end{array}$ & $\begin{array}{l}35 \\
(27.5-51)\end{array}$ & 0.945 & - \\
\hline \multicolumn{5}{|l|}{ Sex } \\
\hline Male & $2(33.3 \%)$ & $4(66.7 \%)$ & 0.597 & - \\
\hline Female & $18(19.2 \%)$ & $76(80.8 \%)$ & & \\
\hline $\begin{array}{l}\text { Time of illness, years } \\
\text { Median (IQR) }\end{array}$ & $5(3.5-10.5)$ & $8(4-14)$ & 0.218 & - \\
\hline Comorbidities & $13(23.6 \%)$ & $42(76.4 \%)$ & 0.315 & - \\
\hline \multicolumn{5}{|l|}{ Systematic commitment to SLE } \\
\hline Articular & $15(20.3 \%)$ & $59(79.7 \%)$ & 0.909 & - \\
\hline Renal & $8(13.3 \%)$ & $52(86.7 \%)$ & $<0.001^{\mathrm{b}}$ & $0.38(0.15-0.91)$ \\
\hline Dermatological & $6(26.1 \%)$ & $17(73.9 \%)$ & 0.391 & - \\
\hline Hematological & $7(33.3 \%)$ & $14(66.7 \%)$ & 0.122 & - \\
\hline Mucocutaneous & $5(29.4 \%)$ & $12(70.6 \%)$ & 0.322 & - \\
\hline Pulmonary & $3(20 \%)$ & $12(80 \%)$ & 1 & - \\
\hline Neurological & $3(20 \%)$ & $12(80 \%)$ & 1 & - \\
\hline Cardiovascular & $1(25 \%)$ & $3(75 \%)$ & 1 & - \\
\hline \multicolumn{5}{|l|}{ Regular treatment for SLE } \\
\hline No treatment & $3(33.3 \%)$ & $6(66.7 \%)$ & 0.378 & - \\
\hline Hydroxychloroquine & $11(14.7 \%)$ & $64(85.3 \%)$ & $<0.001^{\mathrm{b}}$ & $0.20(0.06-0.63)$ \\
\hline Prednisone & $16(22.5 \%)$ & $55(77.5 \%)$ & 0.321 & - \\
\hline Azathioprine & $10(41.7 \%)$ & $14(58.3 \%)$ & $<0.001^{\mathrm{b}}$ & $7.96(2.70-23.43)$ \\
\hline Mycophenolate & $3(13 \%)$ & $20(87 \%)$ & 0.553 & - \\
\hline Methotrexate & $3(37.5 \%)$ & $5(62.5 \%)$ & 0.196 & - \\
\hline Cyclophosphamide & 0 & $5(100 \%)$ & - & - \\
\hline COVID-19 background & $13(33.3 \%)$ & $26(66.7 \%)$ & $<0.001^{\mathrm{b}}$ & $1.68(0.69-4.09)$ \\
\hline $\begin{array}{l}\text { Flare within } 6 \text { months prior to } \\
\text { immunization }\end{array}$ & $8(28.6 \%)$ & $20(71.4 \%)$ & 0.181 & - \\
\hline
\end{tabular}

${ }^{\text {a }} P$ value $<0.05$ indicates statistical significance at $95 \%$ confidence interval

${ }^{b}$ Poisson regression analysis, for a $P$ value adjusted for age, sex, treatment use, history of confirmed COVID-19 and renal involvement, with 95\% confidence interval 
18 after the 1st and 2nd doses, respectively). The most frequent type of reactivation was arthritis (synovitis, phlogosis, functional limitation, accompanied by elevation of acute phase reactants: ESR/CRP) which occurred in $66.6 \%$ in the 1 st dose and $94.4 \%$ in the 2nd dose, followed by skin and scalp involvement (malar erythema and alopecia). Our results are similar to those reported by Izmirly et al. [9] who reported, in a multiethnic study, up to $11.5 \%$ of flare episodes in patients with lupus after SARS-Cov-2 immunization (mainly Pfizer).

Felten et al. [12], in a study of 696 patients with SLE (VACOLUP), described 21 (3\%) self-report episodes of SLE flare (medically confirmed) being mostly musculoskeletal involvement $(90 \%)$. Although our study found a higher proportion of flare episodes (27\%), this could be due to the fact that in VACOLUP, the population was mostly European/USA, the difference in SLE activity in relation to ethnicity is known [17], and that the online survey was a self-report by patients, which could represent a possible underreporting in the number of flares.

For the cases of articular reactivation, given the functional limitation, short courses of increased prednisone doses (or initiation in patients who did not use steroids) were used, with which clinical improvement was evidenced. Similarly, two patients required in-hospital management due to the severity of the condition (lupus pneumonitis and myopericarditis, respectively) which were managed with high doses of corticosteroids (methylprednisolone pulses) and immunosuppressants. After which they were discharged and due to the possible risk of a new episode of flare after the 2 nd vaccine dose, immunization was not recommended.

It should be noted that 10 patients chose not to receive the second dose of the vaccine; among the main reasons were fear of reactivation of SLE, severe reactions after the 1st dose of immunization, and SARS-CoV-2 infection between both doses; one patient had an abortion at 4 weeks of gestation after the 1 st vaccine dose.

The present study has limitations; causality cannot be established in relation to immunization and the flare presented. Likewise, SLEDAI-2 k levels were not compared since not all patients had immunological tests. However, the main strength of the present analysis is that the patients were evaluated prior to immunization, and the episode of SLE reactivation was also established by evaluating (clinically and laboratorial) all cases with suspected flare.

\section{Conclusion}

Although our series describes episodes of reactivation following SARS-CoV-2 immunization (mainly mild), we advise that the COVID-19 immunization process should continue to be a priority for patients with SLE.

\section{Appendix 1}

Table 3 Clinical-epidemiological characteristics of patients with SLE immunized against SARS-CoV-2

\begin{tabular}{ll}
\hline Variables & Total $(N=100)$ \\
\hline Age, years & $35(27.5-49)$ \\
Sex - $n$ (\%) & \\
Female & $94(94 \%)$ \\
Male & $6(6 \%)$ \\
Time of illness (SLE), years & $7.5(4-13)$ \\
Comorbidities* & $55(55 \%)$ \\
Hypothyroidism & $15(15 \%)$ \\
Chronic kidney disease & $13(13 \%)$ \\
APS & $7(7 \%)$ \\
Arterial hypertension & $7(7 \%)$ \\
Rheumatoid arthritis & $6(6 \%)$ \\
Sjögren's syndrome & $5(5 \%)$ \\
Others & $20(20 \%)$ \\
Associated commitment of SLE & \\
Articulate & \\
Renal & $74(74 \%)$ \\
Dermal & $60(60 \%)$ \\
Hematological & $23(23 \%)$ \\
Mucocutaneous & $21(21 \%)$ \\
Pulmonary & $17(17 \%)$ \\
Neurological & $15(15 \%)$ \\
Cardiovascular & $15(15 \%)$ \\
Usual treatment of SLE $\downarrow$ & $4(4 \%)$ \\
Prednisone & \\
Hydroxychloroquine & \\
Azathioprine & $71(71 \%)$ \\
Mycophenolate & $75(75 \%)$ \\
Methotrexate & $24(24 \%)$ \\
Cyclophosphamide & $23(23 \%)$ \\
Leflunomide & $8(8 \%)$ \\
Cyclosporine & $5(5 \%)$ \\
No treatment & $2(2 \%)$ \\
COVID-19 background & $1(1 \%)$ \\
Yes & $9(9 \%)$ \\
No & \\
Hospitalization for COVID-19 & $39(39 \%)$ \\
SLE reactivation in the last 6 months (documented \\
by rheumatologist physician) & $61(61 \%)$ \\
\hline & $3(3 \%)$ \\
& \\
\hline &
\end{tabular}

Data are $n(\%)$ or median (IQR).

*12 patients presented 2 or more comorbidities

$\dagger A P S$ antiphospholipid syndrome

†uvenile idiopathic arthritis (1), avascular necrosis (1), primary biliary cirrhosis (1), diabetes (2), transverse myelitis (1), fibromyalgia (4), pulmonary fibrosis (2), glaucoma (2), asthma (1), aortic stenosis (1), hepatitis C (2), celiac disease (1), pregnancy (1)

$¥ 70$ patients had 2 or more systems affected by SLE

$\$ 72$ patients were taking 2 or more drugs 


\section{Appendix 2}

Fig. 2 Flow chart 130 SLE Patients

8 patients were not vaccinated 4 patients did not agree to participate in the study

2 patients did not authorize follow-up

6 patients discontinued follow-up

100 patients participated in the study

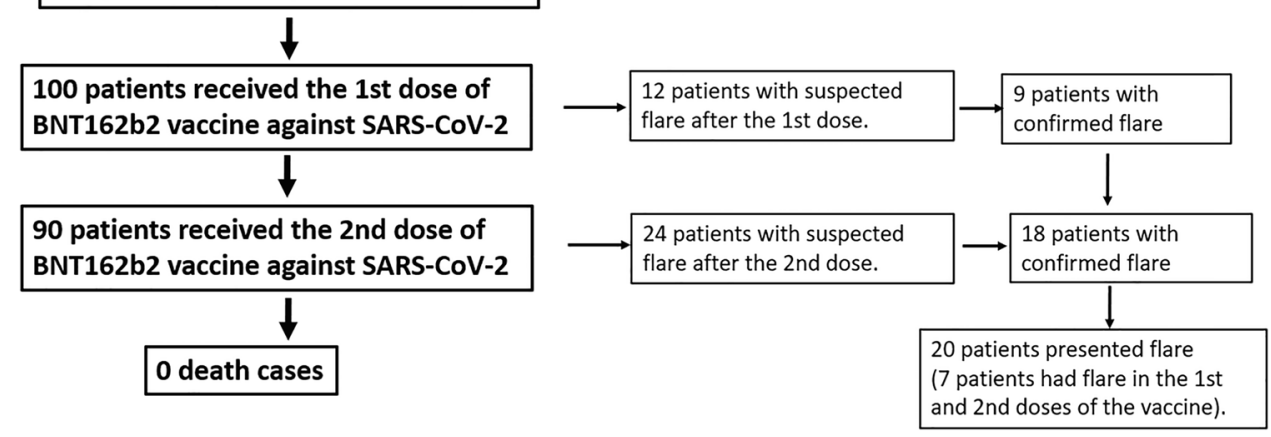




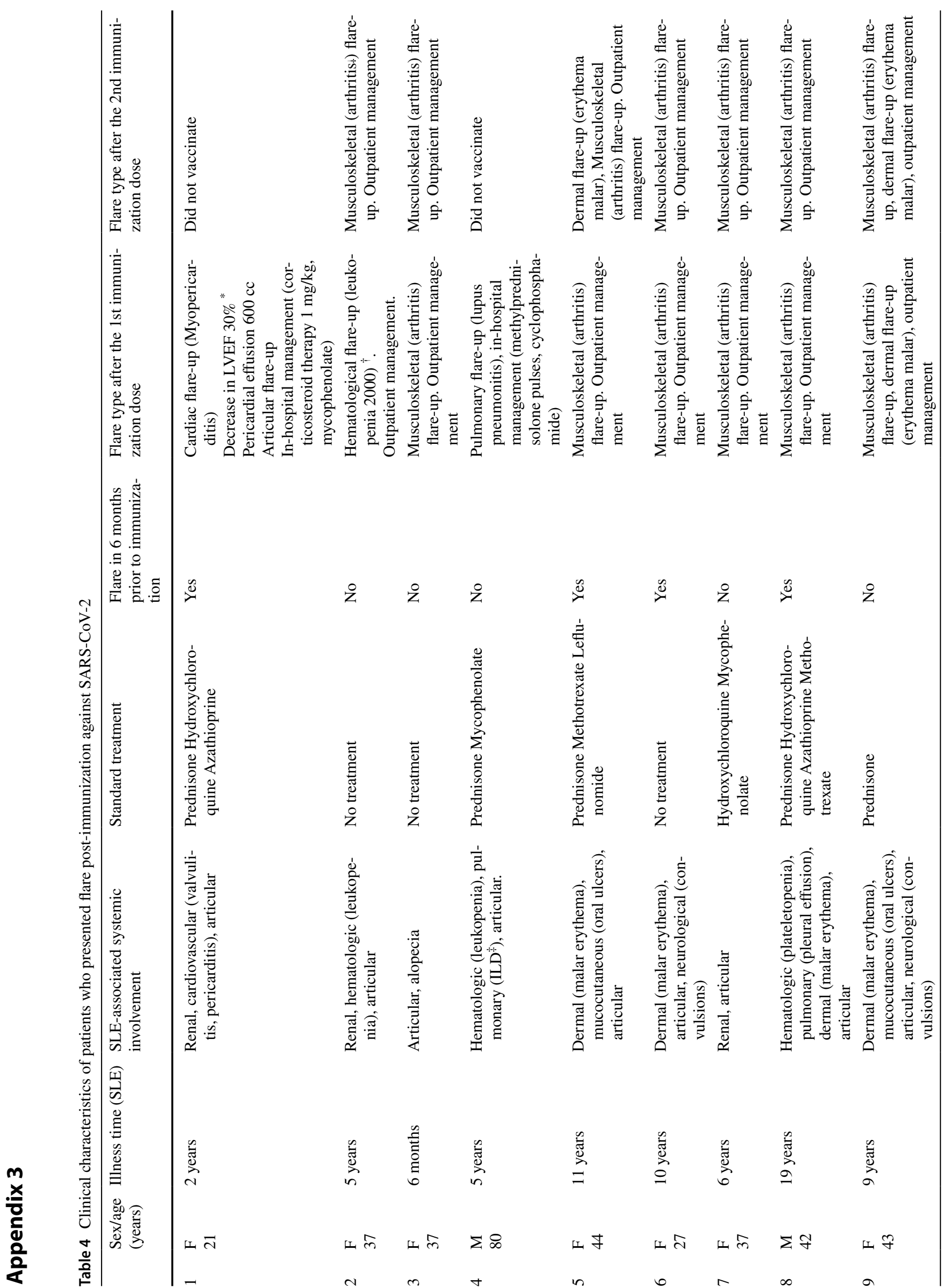




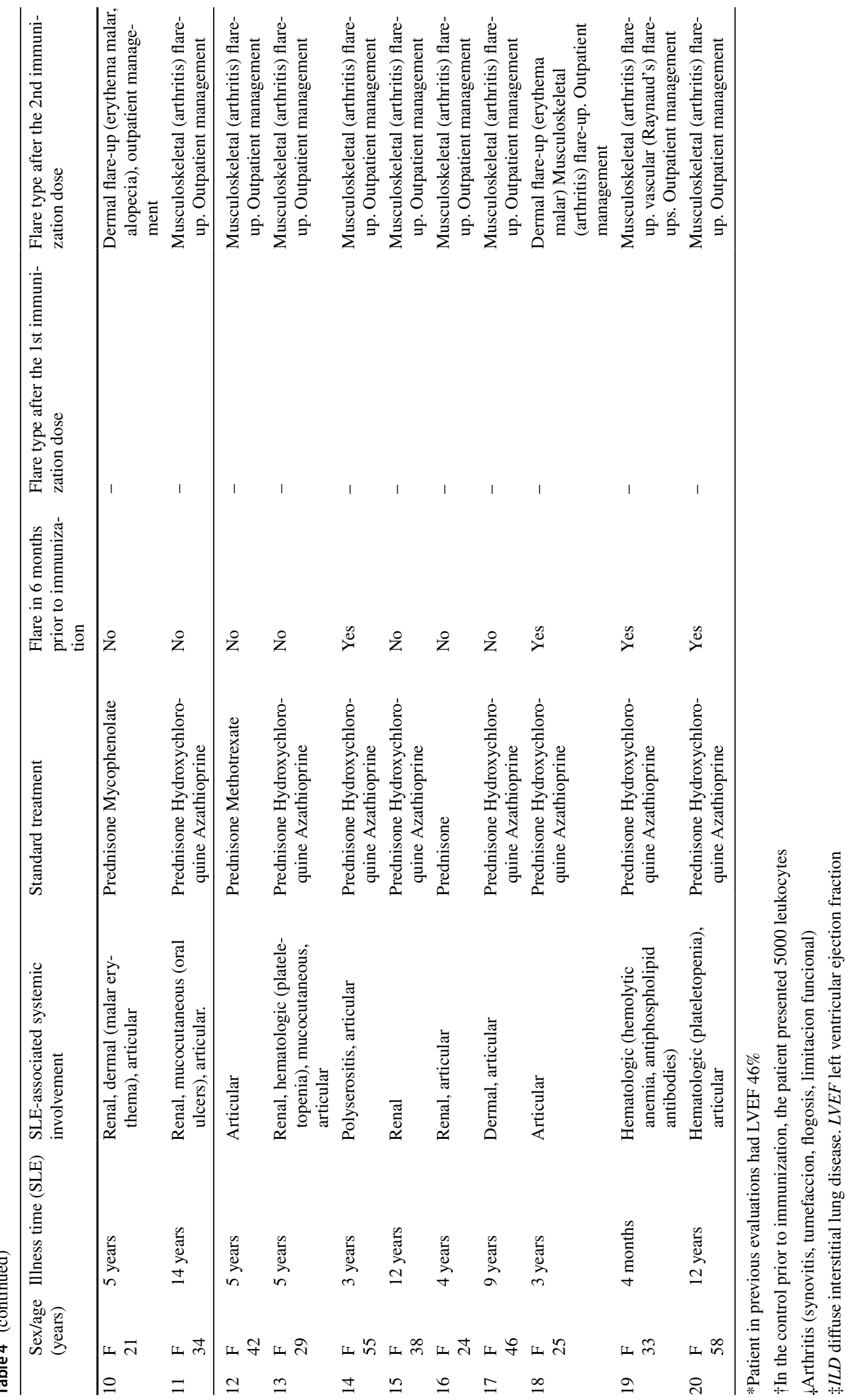


Data availability Only the researchers had access to the database, which was encrypted to maintain the confidentiality of the participants.

Code availability Not applicable.

\section{Declarations}

Ethics approval The present study was approved by the Cayetano Heredia Hospital ethics committee (code 039-2021).

Consent to participate All participants in the present study gave verbal consent.

Conflict of interest The authors declare no competing interests.

\section{References}

1. McGill COVID-19 Vaccine Tracker Team. COVID19 vaccine tracker. https://covid19.trackvaccines.org/vaccines/ Date Accessed 15 Aug 2021

2. Boekel L, Hooijberg F, van Kempen ZLE, Vogelzang EH, Tas SW, Killestein J et al (2021) Perspective of patients with autoimmune diseases on COVID-19 vaccination. Lancet Rheumatol. 3(4):241e243. https://doi.org/10.1016/S2665-9913(21)00037-0

3. Felten R, Dubois M, Ugarte-Gil MF, Chaudier A, Kawka L, Bergier $\mathrm{H}$ et al (2021) Vaccination against COVID-19: expectations and concerns of patients with autoimmune and rheumatic diseases. Lancet Rheumatol 3(4):243-e245. https://doi.org/10.1016/ S2665-9913(21)00039-4

4. Moutsopoulos HM (2021) A recommended paradigm for vaccination of rheumatic disease patients with the SARS-CoV-2 vaccine. J Autoimmun 121:102649. https://doi.org/10.1016/j.jaut.2021. 102649

5. Connolly CM, Ruddy JA, Boyarsky BJ, Avery RK, Werbel WA, Segev DL et al (2021) Safety of the first dose of mRNA SARSCoV-2 vaccines in patients with rheumatic and musculoskeletal diseases. Ann Rheum Dis. https://doi.org/10.1136/annrh eumdis-2021-220231

6. Boyarsky BJ, Ruddy JA, Connolly CM, Ou MT, Werbel WA, Garonzik-Wang JM et al (2021) Antibody response to a single dose of SARS-CoV-2 mRNA vaccine in patients with rheumatic and musculoskeletal diseases. Ann Rheum Dis. https://doi.org/10. 1136/annrheumdis-2021-220289

7. Murdaca G, Orsi A, Spanò F, Puppo F, Durando P, Icardi G et al (2014) Influenza and pneumococcal vaccinations of patients with systemic lupus erythematosus: current views upon safety and immunogenicity. Autoimmun Rev 13(2):75-84. https://doi.org/ 10.1016/j.autrev.2013.07.007
8. Mok CC, Ho LY, Fong LS, To CH (2013) Immunogenicity and safety of a quadrivalent human papillomavirus vaccine in patients with systemic lupus erythematosus: a case-control study. Ann Rheum Dis 72(5):659-664. https://doi.org/10.1136/annrh eumdis-2012-201393

9. Izmirly PM, Kim MY, Samanovic M, Fernandez-Ruiz R, Ohana S, Deonaraine KK (2021) Evaluation of immune response and disease status in SLE patients following SARS-CoV-2 vaccination. Arthritis Rheumatol. https://doi.org/10.1002/art.41937

10. Watad A, De Marco G, Mahajna H, Druyan A, Eltity M, Hijazi N et al (2021) Immune-mediated disease flares or new-onset disease in 27 subjects following mRNA/DNA SARS-CoV-2 vaccination. Vaccines 9(5):435. https://doi.org/10.3390/vaccines9050435

11. Boekel L, Kummer LY, van Dam KPJ, Hooijberg F, van Kempen Z, Vogelzang EH et al (2021) Adverse events after first COVID-19 vaccination in patients with autoimmune diseases. Lancet Rheumatol. 3(8):e542-e545. https://doi.org/10.1016/S2665-9913(21) 00181-8

12. Felten R, Kawka L, Dubois M, Ugarte-Gil MF, Fuentes-Silva Y, Piga M (2021) Tolerance of COVID-19 vaccination in patients with systemic lupus erythematosus: the international VACOLUP study. Lancet Rheumatol. https://doi.org/10.1016/S2665-9913(21) 00221-6

13. Petri M, Orbai AM, Alarcón GS, Gordon C, Merrill JT, Fortin PR et al (2012) Derivation and validation of the systemic lupus international collaborating clinics classification criteria for systemic lupus erythematosus. Arthritis Rheum 64(8):2677-2686. https:// doi.org/10.1002/art.34473

14. Aringer M, Costenbader K, Daikh D, Brinks R, Mosca M, Ramsey-Goldman R (2019) 2019 European league against rheumatism/ American College of Rheumatology Classification Criteria for systemic lupus erythematosus. Arthritis Rheumatol. 71(9):1400 1412. https://doi.org/10.1002/art.40930

15. Ruperto N, Hanrahan LM, Alarcón GS, Belmont HM, Brey RL, Brunetta $P$ et al (2011) International consensus for a definition of disease flare in lupus. Lupus. 20(5):453-462. https://doi.org/10. 1177/0961203310388445

16. Curtis JR, Johnson SR, Anthony DD, Arasaratnam RJ, Baden LR, Bass AR et al (2021) American College of Rheumatology Guidance for COVID-19 vaccination in patients with rheumatic and musculoskeletal diseases - version 1. Arthritis Rheumatol 73(7):1093-1107. https://doi.org/10.1002/art.41734

17. Pons-Estel BA, Catoggio LJ, Cardiel MH et al (2004) The GLADEL multinational Latin American prospective inception cohort of 1,214 patients with systemic lupus erythematosus: ethnic and disease heterogeneity among "Hispanics". Medicine (Baltimore) 83(1):1-17. https://doi.org/10.1097/01.md.0000104742. 42401.e2

Publisher's note Springer Nature remains neutral with regard to jurisdictional claims in published maps and institutional affiliations. 